\title{
Corrigendum: Presynaptic depolarization facilitates neurotrophin-induced synaptic potentiation
}

Lisa Boulanger and Mu-ming Poo

Nat. Neurosci. 2, 346-351 (1999); corrected after print 10 January 2008

In the version of this article initially published, the first author's middle initial was omitted. The correct name should be Lisa M. Boulanger. The error has been corrected in the PDF version of the article.

\section{Corrigendum: Retrograde modulation of presynaptic release probability through signaling mediated by PSD-95-neuroligin}

Kensuke Futai, Myung Jong Kim, Tsutomu Hashikawa, Peter Scheiffele, Morgan Sheng \& Yasunori Hayashi Nat. Neurosci. 10, 186-195 (2007); published online 21 January 2007; corrected after print 15 January 2008

In the version of this article initially published, Figure 5e was duplicated in Figure 5o. The correct Figure 5o is below. The authors regret the error, which has been corrected in the HTML and PDF versions of the article.
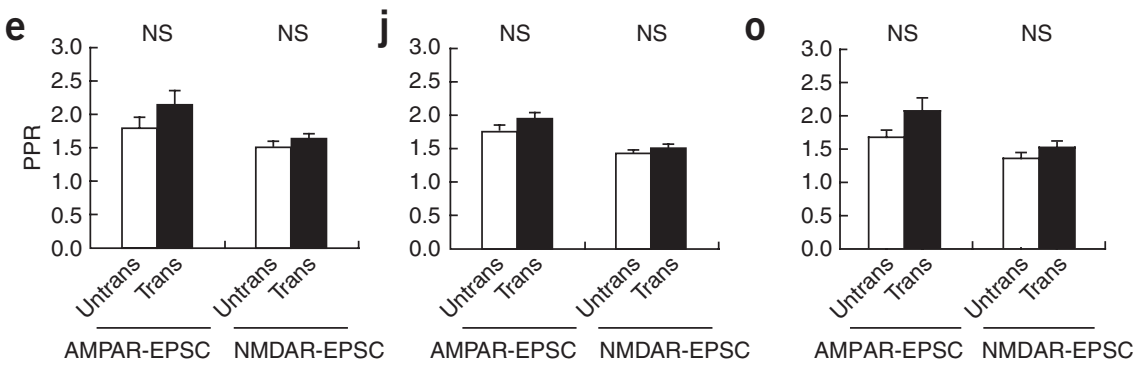

\section{Erratum: MuSK controls where motor axons grow and form synapses}

Natalie Kim \& Steven J Burden

Nat. Neurosci. 11, 19-27 (2008); published online 16 December 2007; corrected after print 11 January 2008

In the version of this article initially published online, several items were omitted from the text. On page 20, left column, the sentence "To determine whether this restricted pattern...regulatory region of the human skeletal $\alpha$-actin (HSA; Fig. 1e)" should read "To determine whether this restricted pattern... regulatory region of the human skeletal $\alpha$-actin gene (HSA; Fig. 1e)". On page 25, left column, the sentence "Muscle is pre-specialized in the central, prospective synaptic region before and independently of innervation, and have led to a revised model of the steps and mechanisms that regulate neuromuscular synapse formation" should read "Muscle is pre-specialized in the central, prospective synaptic region before and independently of innervation, and these findings have led to a revised model of the steps and mechanisms that regulate neuromuscular synapse formation." Finally, on page 19, left column, the sentence "This organizational feature of neurons is essential for forming synapses on appropriate target cells and establishing functional neuronal circuits" should read "This organizational feature of neurons is critical for forming synapses on appropriate target cells and establishing functional neuronal circuits." These errors have been corrected in the HTML and PDF versions of the article. 